\title{
Editorial: special issue on astrodynamics tools and techniques
}

\author{
G. Ortega Hernando ${ }^{1}$ A. Martinez Barrio ${ }^{1}$. C. Yabar Valles ${ }^{1} \cdot$ R. Jehn ${ }^{2}$
}

Published online: 6 February 2018

(c) CEAS 2018

It is a great pleasure for us to present this special issue of the CEAS Space Journal devoted to Astrodynamics Tools and Techniques.

Astrodynamics is a key enabler for space mission design and its tools and techniques are at the core of any spacecraft development and operations.

This special issue of the CEAS Space Journal has been prepared from papers originally coming from the 6th ICATT (International Conference on Astrodynamics Tools and Techniques) and they were selected, revised, and peer reviewed.

The conference was founded in 2001 by ESA and since it has delivered already six editions, every one growing in attendance, importance, and relevance for the vibrant international astrodynamics community.

Thanks to the authors who accepted to submit their papers to the CEAS Space Journal (and thanks to their reviewers), this special number provides an excellent snapshot of the current state of astrodynamics tools and techniques in the fields of orbit propagation, analytical low-thrust methods, inverse dynamics techniques, multi-disciplinary optimisation, finite trust duration manoeuvres, non-Keplerian orbits, aero-stable spacecraft, and new programming languages for astrodynamics.

The first paper (Lara et al.) focuses on the algorithms used in the construction of a semi-analytical propagator for

G. Ortega Hernando

Guillermo.Ortega@esa.int

A. Martinez Barrio

Alvaro.Martinez.Barrio@esa.int

C. Yabar Valles

Celia.Yabar.Valles@esa.int

R. Jehn

Ruediger.Jehn@esa.int

1 Guidance, Navigation, and Control, ESA/ESTEC, Keplerlaan 1, 2201 AZ Noordwijk, ZH, The Netherlands

2 Space Situation Awareness, ESA/ESOC, Robert-Bosch-Str. 5, 64293 Darmstadt, Germany the long-term propagation of highly elliptical orbits (HEO). The software propagates mean elements and includes the main gravitational and non-gravitational effects that may affect common HEO orbits, as, for instance, geostationary transfer orbits or Molniya orbits.

The second paper (Di Carlo et al.) describes CAMELOT: Computational-Analytical Multi-fidelity Low-thrust Optimisation Toolbox. This is a toolbox for the fast preliminary design and optimisation of low-thrust trajectories. It solves highly complex combinatorial problems to plan multi-target missions characterised by long spirals including different perturbations.

The next paper (Virgili et al.) describes an experimental campaign that has been conducted to evaluate the performance of docking manoeuvres of two different guidance and control algorithms based on Model Predictive Control (MPC) and on Inverse Dynamics in the Virtual Domain (IDVD) control.

The next paper (Cremaschi et al.) describes the ASTOS (Analysis, Simulation and Trajectory Optimisation Software) tool that incorporates now a multi-disciplinary optimisation (MDO) and Launcher GNC Simulation and Sizing Tool (LGSST) inside.

The paper after that (Khalil Ben Larbi et al.) derives an advanced control algorithm for far-range formation flight (FF) applications assuming circular reference orbits. The paper focuses on a general impulsive control concept for FF which is then extended to the more realistic case of nonimpulsive thrust manoeuvres. The control concept uses a description of the FF in relative orbital elements (ROE) instead of the classical Cartesian description since the ROE provide a direct access to key aspects of the relative motion and are particularly suitable for relative orbit control purposes and collision avoidance analysis.

The next paper (Evans et al.) describes the tool MONTE: the Mission Analysis, Operations and Navigation Toolkit Environment. MONTE is an astrodynamics toolkit produced by the Mission Design and Navigation Software Group at the Jet Propulsion Laboratory. It provides a single integrated environment for all phases of deep space and Earth orbiting 
missions. Capabilities include trajectory optimisation and analysis, operational orbit determination, flight path control, and $2 \mathrm{D} / 3 \mathrm{D}$ visualisation.

The paper after that (Colagrossi et al.) addresses the dynamics during rendezvous and docking operations with a very large space infrastructure in an Earth-Moon Lagrangian (EML2) Halo orbit. The astrodynamics model takes into account the coupling effects between the orbital and the attitude motion in a Circular Restricted Three-Body Problem environment. The flexibility of the system is included, and the interaction between the modes of the structure and those related with the orbital motion is investigated.

The next paper (Virgili et al.) studies the behaviour of an aero-stable spacecraft. The method described can provide global cross-track and in-track wind measurements. The measurements' accuracy and spatial resolution with respect to the system parameters are analysed and discussed for an ideal one rotational degree-of-freedom case.

The last paper (Eichhorn et al.) provides a study that compares three compiled languages and three interpreted languages to describe and solve astrodynamics problems: FORTRAN, $\mathrm{C}++$, and Java; and Python, MATLAB, and Julia. All six languages are assessed and compared to each other based on their features, performance, and ease-of-use through the implementation of idiomatic solutions to classical astrodynamics problems.

Last but not least, special acknowledgements are due to the Conference Organising Committee.

G. Ortega Hernando

CEAS Space Journal GNC Field Editor 